\title{
Hydrophobic Interactions between Polymeric Carrier and Palmitic Acid-Conjugated siRNA Improve PEGylated Polyplex Stability and Enhance In Vivo Pharmacokinetics and Tumor Gene Silencing
}

Samantha Sarett ${ }^{\dagger}$, Thomas A Werfel ${ }^{\dagger}$, Irene Chandra, Meredith A Jackson, Taylor E Kavanaugh, Madison E Hattaway, Todd D Giorgio, Craig L Duvall*

Department of Biomedical Engineering, Vanderbilt Institute for Nanoscale Science and Engineering, Vanderbilt University School of Engineering, Nashville Tennessee 37232, USA

$\dagger$ These authors contributed equally to this work.

\begin{abstract}
$\underline{\text { Abstract }}$
Formation of stable, long-circulating siRNA polyplexes is a significant challenge in translation of intravenously-delivered, polymeric RNAi cancer therapies. Here, we report that siRNA hydrophobization through conjugation to palmitic acid (siPA) improves stability, in vivo pharmacokinetics, and tumor gene silencing of PEGylated nanopolyplexes (siPA-NPs) with balanced cationic and hydrophobic content in the core (relative to the analogous polyplexes formed with unmodified siRNA, si-NPs). Hydrophobized siPA loaded into the NPs at a lower charge ratio $\left(\mathrm{N}^{+}: \mathrm{P}^{-}\right)$relative to unmodified siRNA, and the siPA-NPs had superior resistance to siRNA cargo unpackaging in comparison to si-NPs upon exposure to the competing polyanion heparin and serum. In vitro, siPA-NPs increased uptake in MDA-MB-231 breast cancer cells (100\% positive cells vs. $60 \%$ positive cells) but exhibited equivalent silencing of the model gene luciferase relative to si-NPs. In vivo in a murine model, the circulation half-life of intravenouslyinjected siPA-NPs was double that of si-NPs, resulting in a $>2$-fold increase in siRNA biodistribution to orthotopic MDA-MB-231 mammary tumors. The increased circulation half-life of siPA-NPs was dependent upon the hydrophobic interactions of the siRNA and the NP core component and not just siRNA hydrophobization, as siPA did not contribute to improved circulation time relative to unmodified siRNA when delivered using polyplexes with a fully cationic core. Intravenous delivery of siPA-NPs also achieved significant silencing of the model gene luciferase in vivo ( $\sim 40 \%$ at 24 hours after one treatment and $\sim 60 \%$ at 48 hours after two treatments) in the murine MDA-MB-231 tumor model, while si-NPs only produced a significant silencing effect after two treatments. These data suggest that stabilization of PEGylated siRNA polyplexes through a combination of hydrophobic and electrostatic interactions between siRNA cargo and the polymeric carrier improves in vivo pharmacokinetics and tumor gene silencing relative to conventional formulations comprising only electrostatic interactions.
\end{abstract}

Keywords: RNA interference, PEGylated Polyplexes, Pharmacokinetics, Hydrophobicity, Breast cancer, Tumor targeting 


\section{Introduction}

Small interfering RNA (siRNA) has the potential to become a transformative class of therapeutics due to its ability to potently and specifically silence expression of genes, including targets considered to be "undruggable" by conventional small molecule inhibitors. However, clinical translation of siRNA therapies has been limited, primarily due to the formidable physiological barriers that must be overcome for siRNA to reach its intracellular site of action ${ }^{1-3}$. When delivered intravenously (e.g., for tumor therapy), siRNA molecules are rapidly cleared through the kidneys ${ }^{4,5}$. If siRNA reaches target cells, it lacks a mechanism to translocate bilayer membranes, limiting both cellular uptake and endosomal escape. To combat these myriad challenges, lipidic and polymeric carrier systems as well as a variety of siRNA conjugates have been developed that feature mechanisms to improve siRNA pharmacokinetics, stability, cellular uptake, release, endosomal escape, and/or site-specific targeting ${ }^{6-19}$. However, clinical efficacy of these delivery systems remains limited, due in large part to a preferential distribution to and systemic clearance through the hepatic and renal systems. As evidenced by therapies currently in advanced clinical trials, the natural targeting of lipid-based nanoparticles to the liver can be leveraged to successfully modulate gene expression in hepatocytes, but delivery of siRNA to other target tissues remains a challenge ${ }^{14,20-24}$. It is thus of high significance to identify systemic siRNA delivery systems that accumulate at other target sites, such as tumors.

Although the magnitude of the enhanced permeability and retention (EPR) effect in spontaneously-formed tumors in humans and large animals is known to be variable, it is accepted that for many tumor types, there is a significant correlation between nanocarrier tumor accumulation and blood circulation persistence (related to avoidance of clearance through organs such as liver and kidney) $)^{25-28}$. Likewise, it has been observed that the magnitude of passive tumor uptake of nucleic acid-based nanopolyplexes is directly related to circulation time ${ }^{29,} 30$. Commonly, lipoplex or polyplex nano-formulations designed for intravenous administration are PEGylated to impart colloidal stability and to reduce opsonization and clearance by the mononuclear phagocyte system (MPS) ${ }^{31-34}$. However, siRNA delivered by polyplexes that are stabilized solely through electrostatic interactions with polyplex core-forming cationic polymers is susceptible to rapid clearance through the kidney. This clearance is due to polyplex disassembly triggered by the competing interactions between the cationic polymer and the polyanionic heparan sulfates of the glomerular basement membrane (GBM). As a result, electrostatically-stabilized or polyion complex nanoparticle formulations impart only minor differences in pharmacokinetics (i.e., blood persistence half-life) relative to free siRNA $\left(t_{1 / 2}\right.$ siRNA $\sim 1-2$ min, $t_{1 / 2}$ siRNA nanoparticles $\left.\sim 3-5 \mathrm{~min}\right)^{4,5,33,35,36}$. While siRNA-encapsulating nanoparticles fabricated through water-in-oil-in water $(\mathrm{W} / \mathrm{O} / \mathrm{W})$ emulsion methods impart significant pharmacokinetic advantages ${ }^{37}$, loading of highly anionic and hydrophilic siRNA into a hydrophobic core nanoparticle formulation is not very efficient, resulting in loss of expensive siRNA during fabrication, exposure of siRNA to potentially damaging organic solvents, and formation of nanoparticles with high weight ratios of carrier polymer(s) relative to siRNA cargo. The aim of the current report was to increase the stability of PEGylated siRNA polyplexes 
against polyanion-induced disassembly, limiting removal of intravenously-delivered polyplexes from the circulation while obviating complex and inefficient siRNA-loaded nanoparticle formulation processes.

Here, we sought to improve pharmacokinetics for tumor applications by developing a PEGylated nanopolyplex formulation that is core-stabilized by both electrostatic and hydrophobic interactions between the polymeric carrier and the siRNA cargo. We recently developed PEGylated, core-loaded siRNA nanopolyplexes (si-NPs) with a combination of both electrostatic and hydrophobic stabilization due to the optimized balance of cationic and hydrophobic content within the polymer block that forms the polyplex $\operatorname{cor}^{38}$. These si-NP formulations comprised unmodified siRNA and the diblock polymer poly(ethylene glycol)- $b$ poly(dimethylaminoethyl methacrylate-co-butyl methacrylate) (PEG- $b$-p(DMAEMA-co-BMA)) with 50 mole percent of both cationic DMAEMA and hydrophobic BMA monomer in the coreforming block (polymer termed "50B"). Relative to the analogous PEG- $b$-p(DMAEMA) diblock polymer (termed " $0 \mathrm{~B}$ " and characterized by a fully cationic core), the $50 \mathrm{~B}$ formulation exhibited improved resistance to disassembly by heparin sulfates, circulation time, and endosomal escape, as well as superior gene silencing bioactivity both in vitro and in vivo. Here, we utilized the 50B polymer for packaging and delivery of hydrophobized siRNA to evaluate the impact of providing both electrostatic and hydrophobic interactions between the polymeric carrier and the siRNA cargo.

To test the hypothesis that a combination of electrostatic and hydrophobic interactions between the 50B polymer and siRNA cargo increases formulation stability and performance, we compared pharmacokinetics and bioactivity of 50B-based nanopolyplexes loaded with unmodified siRNA to those loaded with siRNA conjugated to the hydrocarbon palmitic acid (PA). Conjugation of siRNA to lipid-like moieties (e.g. cholesterol, $\alpha$-tocopherol, and palmitic acid) improves stability and enhances cellular uptake of siRNA by increasing the hydrophobicity of the siRNA molecule ${ }^{15,39,40}$. Furthermore, conjugation to hydrophobic molecules such as cholesterol or palmitic acid (PA) can make siRNA more effective when delivered via polymeric delivery carriers ${ }^{8,12,40-42}$. Similarly, incorporation of hydrophobic components into the polymer carrier has been proven to enhance polyplex stability and cellular uptake and transfection of unmodified nucleic acids ${ }^{16,43-48}$. The Kataoka group has specifically illustrated improvements in stability of a polyplex micelle delivery system via separate investigations into cholesterol modification of the siRNA molecule or micellar components. The approach in the current work is unique in that we investigate the interplay between hydrophobized siRNA and a partially hydrophobic polymer nanocarrier (50B), facilitating hydrophobic as well as electrostatic interactions between cargo and carrier. To isolate the pharmacokinetic significance of hydrophobic interactions between hydrophobized siRNA and the 50B polyplex core versus hydrophobization of each component individually, both the $0 \mathrm{~B}$ (purely cationic) polymer and unmodified siRNA were used as controls for in vivo pharmacokinetics studies. This experimental design elucidates the functional benefit of dual hydrophobization for improving in vivo stability and target gene silencing in an orthotopic triple negative breast cancer (MDA-MB-231) model. 


\section{Experimental Methods}

Materials. Amine-modified single-stranded DNA (modification at 5' end) or RNA (modification at 3' end) and complementary single-stranded Cy5-, Alexa Fluor 488- or Alexa Fluor 546modified DNA or unmodified RNA were all obtained from Integrated DNA Technologies (Coralville, Iowa). The pGreenFire1-CMV plasmid was obtained from System Biosciences (Mountain View, CA), and packaging plasmids pMDLg/pRRE, pRSV-Rev, and pMD2.G were purchased from Addgene (Cambridge, MA). Lipofectamine 2000 and NucBlue Fixed Cell ReadyProbes were purchased from Life Technologies (Grand Island, NY). CytoTox-ONE Homogeneous Membrane Integrity Assay (a lactate dehydrogenase (LDH) assay) was purchased from Promega (Madison, WI). PD10 desalting columns were purchased from GE Healthcare (Waukesha, WI). Quant-iT RiboGreen RNA Assay Kit was purchased from ThermoFisher Scientific (Waltham, MA). All other reagents were purchased from Sigma-Aldrich (St. Louis, $\mathrm{MO})$.

Synthesis of 4-Cyano-4-(ethylsulfanylthiocarbonyl)sulfanylpentanoic Acid (ECT) and PEG-ECT. The RAFT chain transfer agent ECT was synthesized as previously described, and the R-group of the CTA was subsequently conjugated to $\mathrm{PEG}^{7}{ }^{4}{ }^{49}$. Briefly, dicyclohexylcarbodimide ( $4 \mathrm{mmol}, 0.82 \mathrm{~g}$ ) was added to the stirring solution of monomethoxypoly(ethylene glycol) ( $\mathrm{Mn}=5000,2 \mathrm{mmol}, 10 \mathrm{~g})$, ECT (4 mmol, $1.045 \mathrm{~g})$, and DMAP (10 mg) in $50 \mathrm{~mL}$ of dichloromethane. The reaction mixture was stirred for $48 \mathrm{~h}$. The precipitated cyclohexyl urea was removed by filtration, and the dichloromethanane layer was concentrated and precipitated into diethyl ether twice. The precipitated PEG-ECT was washed three times with diethyl ether and dried under vacuum (yield $\sim 10 \mathrm{~g}) .{ }^{1} \mathrm{H}-\mathrm{NMR}\left(400 \mathrm{MHz} \mathrm{CdCl}_{3}\right)$ revealed 91\% substitution of the PEG (data not shown) ${ }^{38}$.

Polymer Synthesis and Characterization. RAFT polymerization was used to synthesize a 50:50 [BMA]:[DMAEMA] copolymer using the PEG-ECT macro-CTA. The target degree of polymerization was 160 , and the monomer plus CTA was $40 \%$ weight per volume in dioxane. The polymerization reaction was carried out at $70^{\circ} \mathrm{C}$ for 24 hours using AIBN as the initiator with a 5:1 [CTA]:[Initiator] molar ratio. A monomer feed ratio of 50:50 mol \% or 0:100 mol \% [BMA]:[DMAEMA] was used (to generate $50 \mathrm{~B}$ and $0 \mathrm{~B}$ respectively), and a double alumna column was utilized to remove inhibitors from DMAEMA and BMA monomers prior to polymerization. The reactions were stopped by removal from heat and exposure of the polymerization solution to air. The resulting polymers were precipitated into a co-solvent of $90 \%$ pentane and $10 \%$ diethyl ether. The isolated polymers were vacuum-dried, redissolved in water, further purified by dialysis for 24 hours, and lyophilized. Polymers were characterized for composition and molecular weight by ${ }^{1} \mathrm{H}-\mathrm{NMR}$ spectroscopy (Bruker $400 \mathrm{MHz}$ spectrometer equipped with a 9.4 $\mathrm{T}$ Oxford magnet). Absolute molecular weight and polydispersity of the polymers was determined using DMF mobile phase gel permeation chromatography (GPC, 
Agilent Technologies, Santa Clara, CA, USA) with inline Agilent refractive index and Wyatt miniDAWN TREOS light scattering detectors (Wyatt Technology Corp., Santa Barabara, CA, USA).

Oligonucleotide-PA Synthesis and Characterization. Single-stranded amine-modified oligo was reacted with 100-fold molar excess of PA $N$-hydroxysuccinimide ester pre-dissolved at 40 $\mathrm{mM}$ in $N, N$-dimethylformamide (DMF). The reaction was carried out for 18 hours at room temperature in $45 \%$ water, $45 \%$ isopropyl alcohol, and $10 \%$ DMF. The oligo-PA was purified by reversed-phase HPLC using a Clarity Oligo-RP column (Phenomenex, Torrence, CA) under a linear gradient from $95 \%$ water (50 mM triethylammonium acetate), $5 \%$ methanol to $100 \%$ methanol. The conjugate molecular weight was confirmed using MALDI-TOF mass spectrometry (Voyager-DE STR Workstation, Grand Island, NY) using $50 \mathrm{mg} / \mathrm{mL} \mathrm{3-}$ hydroxypicolinic acid in $50 \%$ water, $50 \%$ acetonitrile with $5 \mathrm{mg} / \mathrm{mL}$ ammonium citrate as a matrix. The yield of the oligo-PA was quantified based on absorbance at $260 \mathrm{~nm}$. The purified oligo-PA was annealed to its complementary strand to generate Cy5-, Alexa Fluor 488- or Alexa Fluor 546-modified DNA-PA or siPA. Conjugation and annealing was also confirmed via agarose gel electrophoresis.

Assembly and Characterization of siRNA- or siPA-Loaded Polyplex NPs. Polyplex NPs loaded with siRNA (si-NPs) or siPA (siPA-NPs) were made by mixing $\mathrm{pH} 4.0$ stock solutions of 50B polymer (10 $\mathrm{mM}$ buffer, $3.33 \mathrm{mg} / \mathrm{mL}$ polymer) and siRNA $(50 \mu \mathrm{M})$ at $\mathrm{N}: \mathrm{P}$ ratios of $1,2,5$, 7,10 , or 20 . Control polyplexes comprising the $0 \mathrm{~B}$ polymer (termed si-0B-NPs and siPA-0BNPs) were made according to the same procedure. The final charge ratio was calculated as the molar ratio of cationic amines on the DMAEMA $(50 \%$ are assumed to be protonated at physiologic $\mathrm{pH}$ ) to the anionic phosphates on the siRNA/siPA. After mixing, these solutions were diluted 5-fold to $100 \mu \mathrm{L}$ with $\mathrm{pH} 8.0$ phosphate buffer $(10 \mathrm{mM})$ to adjust the final $\mathrm{pH}$ to 7.4. After mixing, samples were incubated for $30 \mathrm{~min}$, and $100 \mathrm{ng}$ of siRNA/siPA for each sample was loaded onto a $2 \%$ agarose gel containing ethidium bromide to assess siRNA/siPA packaging efficiency. The gels were run at $100 \mathrm{~V}$ for $35 \mathrm{~min}$ and imaged with a UV transilluminator. Hydrodynamic diameter and zeta potential of the polyplex NPs at the N:P ratios described above were measured in triplicate using dynamic light scattering (DLS) (Malvern Zetasizer Nano ZS, Malvern, UK). DLS measures were used to evaluate salt stability of polyplex $\mathrm{NPs}$; concentrated $\mathrm{NaCl}$ solution was added to si-NP or siPA-NP solutions in $10 \mathrm{mM}$ phosphate buffer to yield final $\mathrm{NaCl}$ concentrations of $0,0.1,0.5$, or $1 \mathrm{M}$ where the final solution was $80 \%$ phosphate buffer by volume. For the cell uptake studies (where no functional effects were studied) DNA and DNA-PA was used as a model molecule for siRNA and siPA, respectively. Hereafter, NPs loaded with these molecules are referred to as si-NPs and siPA-NPs to avoid confusion. 
Cell Culture. Human epithelial breast cancer cells (MDA-MB-231) were cultured in Dulbecco's modified Eagle's medium (DMEM, Gibco Cell Culture, Carlsbad, CA) supplemented with 10\% fetal bovine serum (FBS, Gibco) and $0.1 \%$ gentamicin (Gibco).

Production of Stable Luciferase-Expressing MDA-MB-231s. To produce lentivirus, the pGreenFire1-CMV plasmid and packaging plasmids pMDLg/pRRE, pRSV-Rev, and pMD2.G were transfected into HEK-293Ts using Lipofectamine 2000. Media was changed after 24 hours and supernatant containing lentivirus was collected at 48 and 72 hours. Viral supernatant was added directly to MDA-MB-231s with $6 \mu \mathrm{g} / \mathrm{mL}$ polybrene. Media was changed after 24 hours. Lentiviral transduction and was confirmed by GFP expression as analyzed by flow cytometry (BD LSR II Flow Cytometer, San Jose, CA). This was followed by selection with $8 \mu \mathrm{g} / \mathrm{mL}$ puromycin for two weeks to eliminate non-transduced cells.

Cellular Uptake. MDA-MB-231s were seeded at 30,000 cells/well in 24-well plates and allowed to adhere overnight. After adhering, cells were treated with $100 \mathrm{nM}$ Alexa Fluor 488labeled si-NPs or siPA-NPs in $10 \%$ serum for 24 hours. Lipofectamine was used as a positive control (with treatment at $25 \mathrm{nM}$ to minimize toxicity). After $24 \mathrm{~h}$, media with treatments was removed, cells were washed with PBS (-/-), trypsinized $(0.25 \%)$, transferred to microcentrifuge tubes, and centrifuged at $420 \mathrm{xG}$ for $7 \mathrm{~min}$ to pellet the cells. Pellets were re-suspended in $0.4 \mathrm{~mL}$ PBS(-/-) with $0.04 \%$ trypan blue to quench extracellular fluorescence and monitored by FACS (FACSCalibur, BD Biosciences, Franklin Lakes, NJ, USA) at excitation wavelength of $488 \mathrm{~nm}$ and emission wavelength of $519 \mathrm{~nm}$ to quantify intracellular delivery.

In Vitro Gene Silencing. MDA-MB-231s were treated with si-NPs or siPA-NPs; the siRNA was either designed against the luciferase gene (luc siRNA) or was a scrambled sequence (scr siRNA). Cells were seeded at 2,000 cells/well in 96-well black-walled plates and allowed to adhere overnight. Cells were then treated in $10 \%$ serum for 24 hours at a dose of $100 \mathrm{nM}$ siRNA. After $24 \mathrm{~h}$, media was replaced with luciferin-containing media $(150 \mu \mathrm{g} / \mathrm{mL})$ before imaging with an IVIS Lumina III imaging system (Caliper Life Sciences, Hopkinton, Massachusetts) every 24 hours for 10 days. Fresh low serum media (2\% FBS) was replaced after each imaging session, and cells were passaged every 3 days. Growth in low serum was used to reduce the confounding influence of proliferation and allowed more direct investigation of gene silencing longevity. To evaluate treatment cytotoxicity, scr siRNA si-NP treatments were removed at 24 hours and cellular bioluminescence was quantified on a Lumina III IVIS (Caliper Life Sciences, Hopkinton, Massachusetts) and compared to no treatment as a measure of relative cell number.

Comparison of siRNA and siPA Polyplex Stability. NPs were loaded with Förster Resonance Energy Transfer (FRET, using Alexa Fluor 488 and Alexa Fluor 546) pair-labeled doubledstranded 23mers (FRET-NPs). Fluorescent intensity was measured using a microplate reader (Tecan Infinite F500, Männedorf, Switzerland) with an excitation wavelength of $488 \pm 5 \mathrm{~nm}$. 
Alexa Fluor 488 emission was collected at $519 \pm 5 \mathrm{~nm}$, and Alexa Fluor 546 emission was obtained at $573 \pm 5 \mathrm{~nm}$. FRET was calculated as a ratio of the fluorescent intensity as follows:

$\mathrm{FRET}=I 5731519$

Because siRNA decomplexation by heparan sulfate-containing glomerular basement membrane in the kidney is a primary cause for rapid systemic clearance of polycation-siRNA nanoparticles, the stability of FRET-NPs was measured in the presence of 2 to $100 \mathrm{U} / \mathrm{mL}$ of heparin sodium salt in $\mathrm{DPBS}^{4,5}$. The fluorescence emission was measured over time following addition of heparin sodium salt. The heparin concentration at which the FRET signal was reduced 50\% (EC50) for siRNA and siPA polyplexes was calculated as according to the following equation where $\mathrm{y}$ is the FRET ratio, $\mathrm{x}$ is the heparin concentration, and $\mathrm{b}$ is a fit parameter.

$y=-11+x E C 50 b+1$

The same assay was performed in the presence or absence of 10, 40, and 50\% of FBS as well. In this study, si-NPs and siPA-NPs were prepared as described above and incubated with either FBS or an equal volume of PBS. FRET was calculated according to the equation above, and \%FRET was calculated at each time point by dividing the FRET ratio of FBS-treated NPs by PBS-treated controls. The Quant-iT RiboGreen RNA Assay Kit was used to quantify the amount of unpackaged siRNA before and after addition of $100 \mathrm{U} / \mathrm{mL}$ heparin for si-NPs, siPA-NPs, si0B-NPs, and siPA-0B-NPs.

Blood Plasma Pharmacokinetics. Fluorescent (Cy-5-labeled) si-NPs and siPA-NPs were formed at an N:P ratio of 10:1 As a comparison, siRNA and siPA were loaded into $0 \mathrm{~B}$ at a ratio of 10:1. NPs were injected into the tail vein of CD-1 mice (4-6 weeks old, Charles Rivers Laboratories, Wilmington, MA, USA) at $1 \mathrm{mg} / \mathrm{kg}$. Blood was collected retro-orbitally at $5 \mathrm{~min}$ and 10 min post-injection, not exceeding two collections per animal. After $20 \mathrm{~min}$, animals were sacrificed, and blood was immediately collected via cardiac puncture. Blood samples were centrifuged at $2000 \mathrm{G}$ for $5 \mathrm{~min}$ and $5 \mu \mathrm{L}$ of plasma was taken from the supernatant and diluted into $95 \mu \mathrm{L}$ PBS (-/-). Fluorescence was measured and quantified on an IVIS Lumina III imaging system (Xenogen Corporation, Alameda, CA, USA) at excitation wavelength of $620 \pm 5 \mathrm{~nm}$ and emission wavelength of $670 \pm 5 \mathrm{~nm}(\mathrm{n}=6)$. A standard curve was generated by measuring the fluorescence of the initial fluorescent polyplex solution in PBS (-/-) over the range of $200 \%$ to $1.5 \%$ of the injected dose. The standard curve was utilized in order to calculate the percent of injected dose in each blood sample, and the calculated values were used to determine siRNA concentration in the plasma at each time point as well as area under the curve (AUC) values (see Table S1 for equations).

Biodistribution in Tumor-bearing Mice. Athymic nude female mice (4-6 weeks old, Jackson Laboratory, Bar Harbor, ME, USA) were injected in each mammary fat pad with 1 x $10^{6}$ MDAMB-231 cells in DMEM:Matrigel (50:50). After 17 days, tumor-bearing mice were injected via the tail vein with $1 \mathrm{mg} / \mathrm{kg}$ (nucleic acid dose) of fluorescent siNPs or siPA-NPs. After 20 minutes, animals were sacrificed and the organs of interest (heart, lungs, liver, spleen, kidneys, 
and tumors) were excised. The fluorescence intensity in the organs was quantified on an IVIS Lumina III imaging system (Xenogen Corporation, Alameda, CA, USA) at excitation wavelength of $620 \pm 5 \mathrm{~nm}$ and emission wavelength of $670 \pm 5 \mathrm{~nm}(\mathrm{n}=3$ animals, $\mathrm{n}=6$ tumors).

Target Gene Silencing After Intravenous (I.V.) Injection. Athymic nude female mice (4-6 weeks old, Jackson Laboratory, Bar Harbor, ME, USA) were injected in each mammary fat pad with 1 × $10^{6}$ MDA-MB-231 cells in DMEM:Matrigel (50:50). After 17 days, tumor-bearing mice were injected i.p. with luciferin substrate $(150 \mathrm{mg} / \mathrm{kg})$ and imaged for bioluminescence on an IVIS Lumina III imaging system (Xenogen Corporation, Alameda, CA, USA) 20 minutes postinjection. Next, the mice were injected via the tail vein with $1 \mathrm{mg} / \mathrm{kg}$ (siRNA dose) NPs containing either luc siRNA / siPA, a scr siRNA / siPA, or saline. Mice were imaged and treated at days 17 and 18 following tumor cell inoculation and imaged on day 19. Relative luminescence was determined by measuring the raw luminescent intensity of each tumor on each day and comparing to the initial signal at day $17(\mathrm{n}=10$ tumors per group).

Acute Toxicity in Liver and Kidney. Tumor-bearing mice used for in vivo luciferase silencing studies were sacrificed on day 20 following tumor cell inoculation (and following treatment with $1 \mathrm{mg} / \mathrm{kg}$ siRNA on days 17 and 18). Blood was collected by cardiac puncture and then centrifuged at $2000 \mathrm{G}$ for $5 \mathrm{~min}$. Then, plasma was harvested and tested by the Vanderbilt Translational Pathology Shared Resource (TPSR) for systemic levels of alanine aminotransferase (ALT), aspartate aminotransferase (AST), and blood urea nitrogen (BUN).

Statistical Methods. The treatment groups were statistically compared using a one way ANOVA test coupled with a Tukey means comparison test; a p-value $<0.05$ was deemed representative of a significant difference between groups. For all data shown, the arithmetic mean and standard error are reported, and the sample size (n) is indicated.

Ethics Statement. The animal studies were conducted with adherence to the guidelines for the care and use of laboratory animals of the National Institutes of Health (NIH). All experiments with animals were approved by Vanderbilt University's Institutional Animal Care and Use Committee (IACUC).

\section{Results and Discussion}

Polymer and siPA Conjugate Synthesis. A previously reported RAFT polymerization scheme was used to synthesize the 50B polymer from a $5 \mathrm{kDa}$ PEG-ECT macro-CTA ${ }^{38}$. The scheme is desirable for its simplicity and scalability, and it consistently yields polymers at target molecular weight (MW) with low polydispersity index (PDI). The 50B polymer used here was synthesized from the macro-CTA with a final degree of polymerization (DP) of 152 (Target DP: 160) and PDI of 1.03 as determined by ${ }^{1} \mathrm{H}-\mathrm{NMR}$ and GPC, respectively (Figure 1). The RAFTpolymerized block monomer composition and MW were quantified by ${ }^{1} \mathrm{H}-\mathrm{NMR}$ using 
characteristic peaks from PEG (-O- $\left.\mathrm{CH}_{2} \mathrm{CH}_{2^{-}}, \delta 3.65 \mathrm{~s}\right), \mathrm{BMA}\left(-\mathrm{O}-\mathrm{CH}_{2} \mathrm{CH}_{2^{-}}, \delta 3.95 \mathrm{~s}\right)$, and DMAEMA (-O- $\mathrm{CH}_{2} \mathrm{CH}_{2-}, \delta 4.05 \mathrm{~s}$ ), showing 49:51 (BMA:DMAEMA) mol\% ratio in the polyplex core-forming block and total MW of 27,800 Da (including 5kDa PEG). The control 0B polymer was synthesized by the same 5route and had a $110 \mathrm{DP}, 1.16 \mathrm{PDI}, 0: 100 \mathrm{~mol} \%$ ratio, an0 d 22,300 Da MW (data not shown).

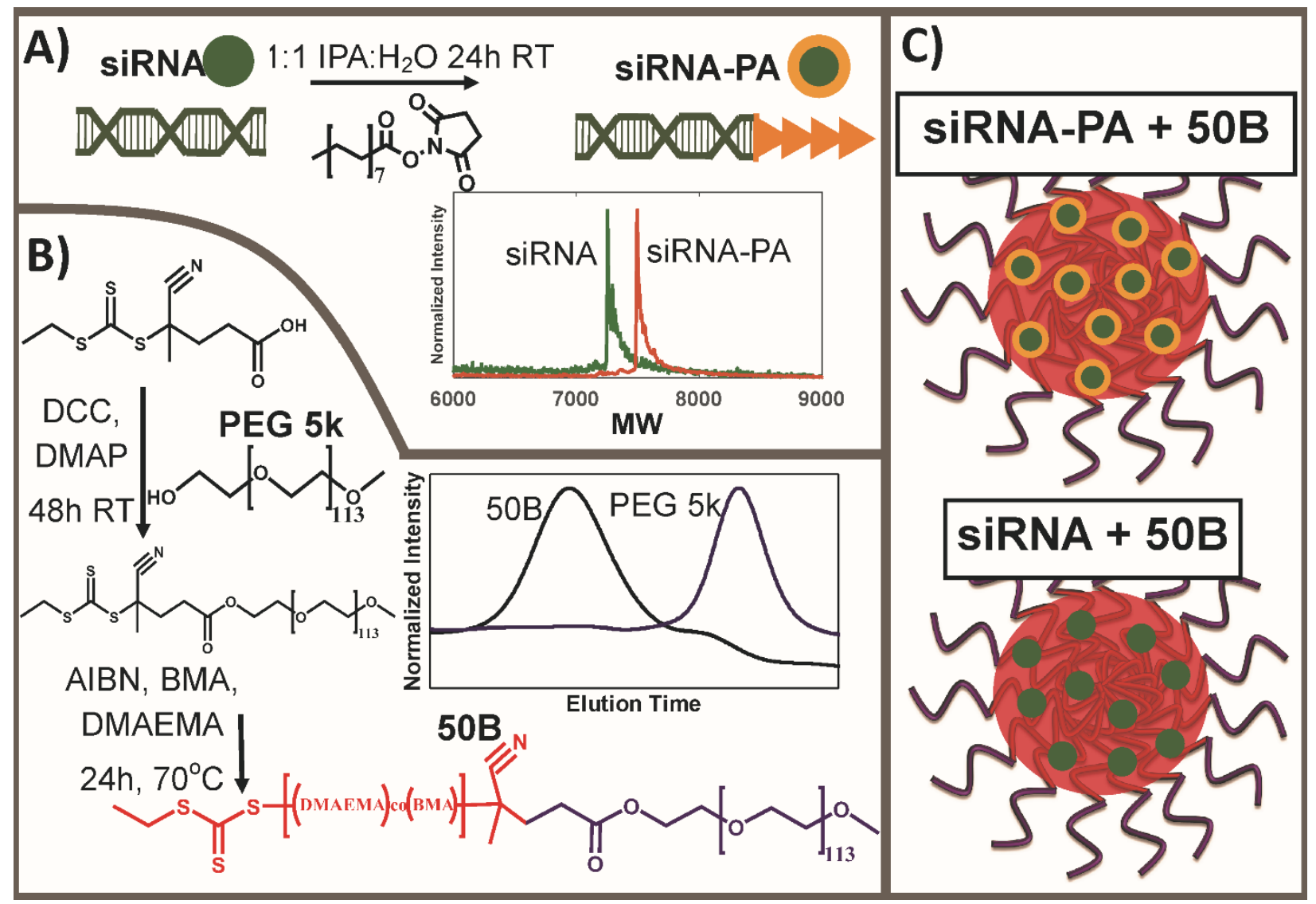

Figure 1: A) Synthesis of siPA and product confirmation via MALDI-TOF. B) Synthesis of 50B and GPC analysis. C) Schematic of core-loaded siPA-NP and si-NP polyplexes.

Synthesis of siPA-NPs. Single-stranded DNA or RNA was successfully conjugated with PA in a one-step reaction and purified from the reactants via HPLC. Isolation of the desired products was confirmed by MALDI-TOF analysis (Figure 1) and also by shift upward of the free siPA band in comparison to the unmodified siRNA band in a gel retardation assay (Figure 2). 


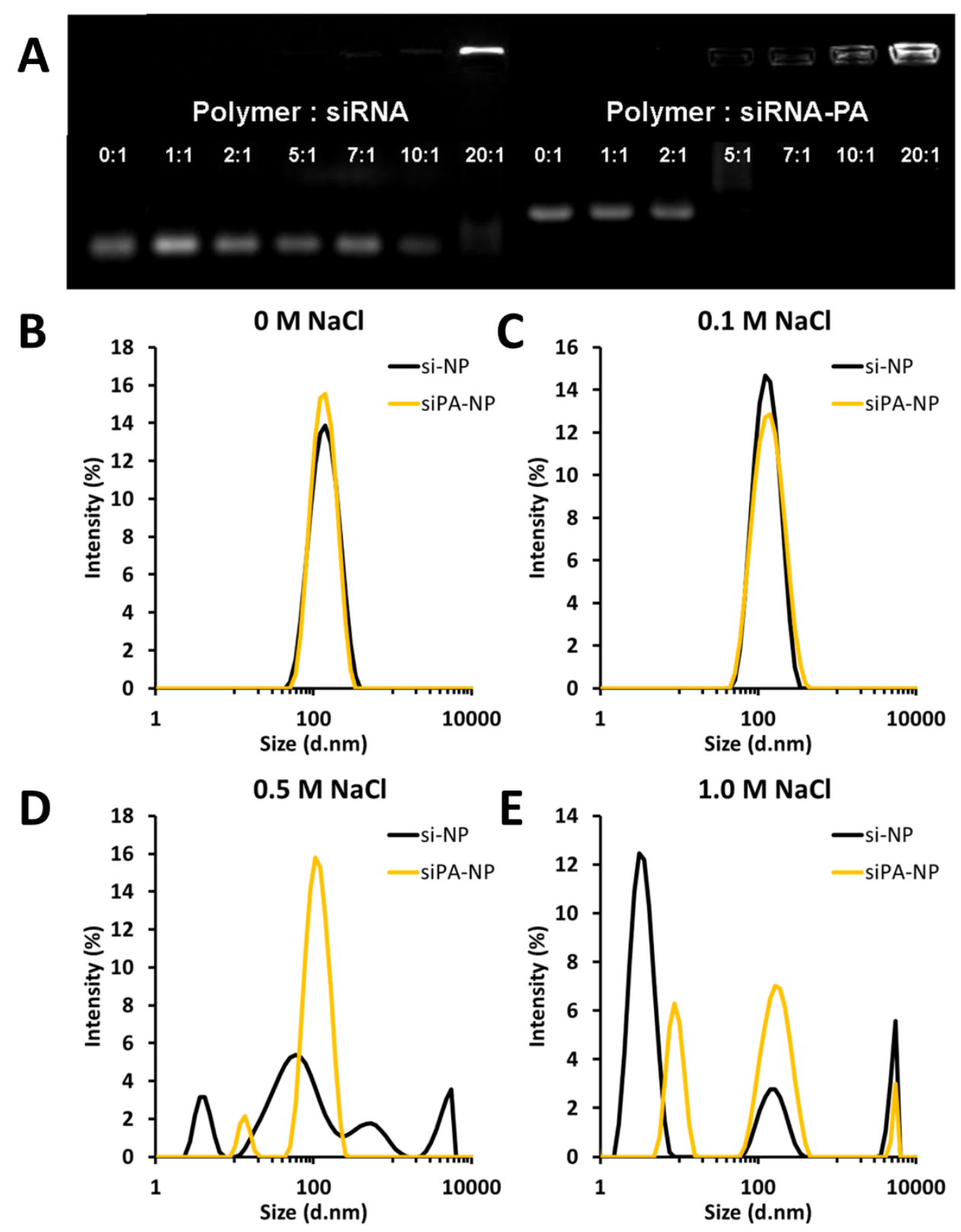

Figure 2: siPA is packaged more efficiently and stably with 50B polymer than unmodified siRNA. A) As evaluated by gel retardation assay, siPA loads fully at a lower N:P ratio than unmodified siRNA; note that the upward shift of siPA compared to siRNA also confirms PA conjugation. B-D) As evaluated by dynamic light scattering, siPA-NPs are more stable to elevated salt concentrations (approximately $25 \mathrm{~nm}$ smaller) than si-NPs at $\mathrm{N}: \mathrm{P}=10: 1$.

Characterization of si-NPs/siPA-NPs. Unmodified siRNA completely loaded into the PEGylated nanopolyplexes at an N:P ratio of 20:1, while siPA was fully loaded at a ratio of 5:1, as characterized by gel retardation assays (Figure 2A). This result suggests that hydrophobization of the siRNA molecule enhances interactions with 50B and improves efficiency of loading into NPs. DLS measurements reveal that siPA-NPs are of equivalent size and exhibit enhanced stability to elevated salt concentration relative to si-NPs (Figure $2 \mathrm{~B}$ and S2). The zeta potential 
does not differ significantly between siPA-NPs and si-NPs, with each displaying a slight negative charge which is optimal for intravenous administration (data not shown).

This result is in agreement with previous reports from our group and others showing that lipid-modified siRNA loads more efficiently into nanocarrier systems with completely electrostatically-driven siRNA complexation mechanisms ${ }^{8,42,50}$. However, the siPA-NP system is unique in that it is the first to elucidate the importance of hydrophobic interactions between lipid-modified siRNA and hydrophobized cationic polymer components. We posit that the hydrophobization of the siRNA stabilizes the nanoparticles by introducing interaction between the lipophilic moieties on different siRNA molecules and, unique to the siPA-NPs, with the hydrophobic BMA monomer which is $\sim 50 \mathrm{~mol} \%$ of the core-forming block of the carrier polymer in the current system. This overall result indicates that a combination of electrostatic and hydrophobic interactions between siPA and 50B improved its loading efficiency and stability in the presence of competing polyelectrolytes (akin to those encountered in systemic administration), motivating further characterization of the stability and function of siPA-NPs in biological contexts.

In vitro validation of si-NPs/siPA-NPs. After establishing the improved loading efficiency of siPA relative to unmodified siRNA, we characterized the performance of si-NPs and siPA-NPs in vitro (Figure 3). Evaluation of the cytotoxicity of each demonstrated greater than $80 \%$ cell viability at all N:P ratios examined after $24 \mathrm{~h}$ (Figure 3A), with a trend toward decreased cell number for higher N:P ratios. This result confirms that the nanopolyplex system is generally well-tolerated but also emphasizes the translational significance of reducing the amount of polymer necessary to achieve a therapeutic effect, for example through improved siRNA loading and delivery using a lower N:P ratio. 

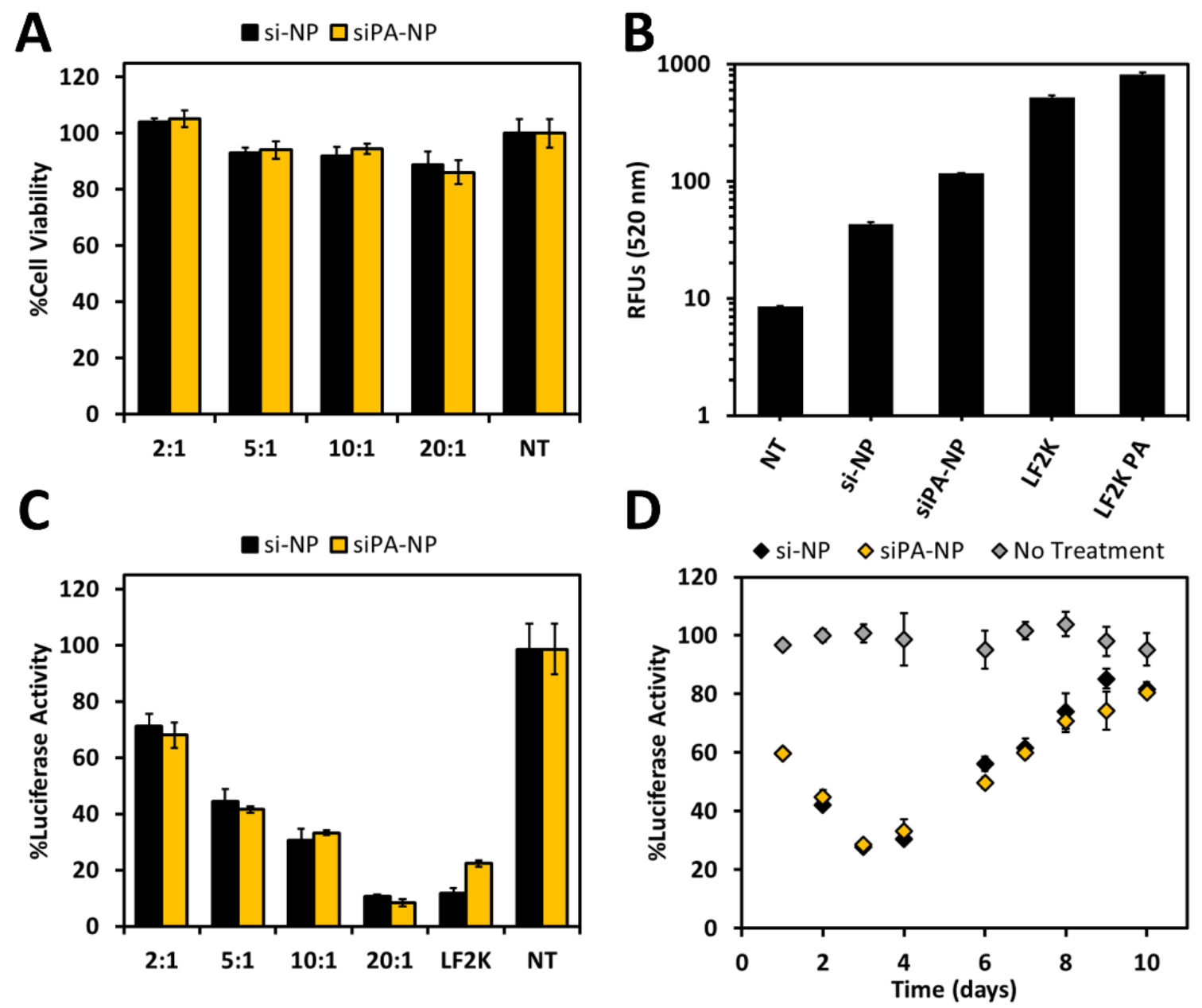

Figure 3: In vitro characterization of siPA-NPs vs. si-NPs. A) Both formulations exhibit $>80 \%$ cell viability at all $\mathrm{N}: \mathrm{P}$ ratios investigated as evaluated by percent difference in luciferase signal from that of no treatment $(\mathrm{n}=4)$. B) siPA-NPs are internalized by cells $\sim 2$-fold more than si-NPs after 24 hours of treatment $(\mathrm{N}: \mathrm{P}=10: 1, \mathrm{n}=3)$. C) siPA-NPs and si-NPs exhibit increasing luciferase silencing at higher N:P ratios but are not significantly different from each other. $(n=4)$. D) Both siPA-NPs and si-NPs show prolonged luciferase silencing (over 10 days) at an N:P ratio of 10:1 $(n=3)$. For $C)$ and $D)$, all treatment groups are normalized to analogous scrambled siRNA controls to account for treatment effect on cell viability; no treatment is averaged each day by measuring luminescent signal of untreated cells $(n=3)$.

Next, the cellular uptake of siPA-NPs was compared to si-NPs. After treatment with siPA-NPs, nearly $100 \%$ of cells were positive for the fluorescently-tagged nucleic acid (Figure S3). This was equivalent to the percentage positive cells observed after treatment with Lipofectamine ${ }^{\circledR} 2000$, a commercial transfection reagent. The corresponding treatment of si-NPs resulted in a cell population $60 \%$ positive (Figure S3), revealing that PA conjugation increased NP cellular internalization. This is corroborated by evaluation of the mean fluorescent intensity of treated cells, which was approximately 2-fold higher for siPA-NPs compared to si-NPs (Figure 3B). Many physicochemical and biological factors such as particle size ${ }^{51,52}$, surface charge $^{51}$, shape $^{53}$, PEG density ${ }^{54}$, particle elasticity ${ }^{55}$, internalization and trafficking route ${ }^{56,57}$, 
etc., can contribute to differences in particle uptake. In our studies, the most obvious difference between the si-NPs and siPA-NPs was enhanced stability of siPA-NPs in the presence of serum (Figure S4). Therefore, it is likely that increased siPA-NP stability in the presence of serum contributes to the observed improvement in uptake, although this result may be multifactorial.

In in vitro gene knockdown screens, the si-NPs and siPA-NPs both exhibited potent and sustained silencing in MDA-MB-231 breast cancer cells (Figure 3C, D). For each, a higher level of gene silencing was observed as the N:P ratio was increased. Silencing between si-NPs and siPA-NPs did not differ significantly across the N:P ratios screened, despite the small increase in cell uptake observed for siPA-NPs. This could be due to the enhanced stability of the siPA-NPs, which may impede siRNA unpackaging from nanopolyplexes upon cellular internalization ${ }^{58}$. Although increased polyplex stability may limit siRNA intracellular bioavailability in vitro, this potentially negative impact is expected to be outweighed by the benefit gained in vivo by increasing circulation half-life and cell uptake. Also of note is the prolonged silencing effect observed in this nanoparticle system, using both unmodified siRNA and siPA (Figure 3D). A potential factor in this sustained effect in vitro is the inherent endosomolytic capability of the 50B polymer carrier; by avoiding endosomal degradation and/or trafficking from the cell, endosomolytic carriers have been shown to elicit desirable durability of therapeutic action ${ }^{59}$. The prolonged effect of si-NPs and siPA-NPs in vitro, with significant silencing out to 10 days posttreatment, suggests that this delivery system achieves a sustained effect that would minimize the need for repeat dosing.

Pharmacokinetics and Biodistribution. Targeting siRNA nanoparticles to cancer targets such as solid tumors in vivo is contingent upon the ability to avoid rapid clearance by the liver (phagocytosis) and kidneys (polyanionic disassembly), which extends circulation time and consequently passive tumor uptake by the EPR effect. Disassembly in the kidney, leading to clearance through the urine, is especially detrimental to siRNA polyplex circulation time ${ }^{4}$. We showed previously that 50B-based si-NPs, which have balanced cationic and hydrophobic character in the polymeric block that forms the polyplex core, are more resilient to heparin sulfate disassembly and have longer circulation than strictly cationic analogues ${ }^{38}$. These si-NPs are used as a benchmark to compare siPA-NPs which incorporate hydrophobicity into both the polymer backbone and siRNA molecule.

The siPA-NPs have increased stability upon exposure to heparin compared to si-NPs as monitored by \%FRET over time. Neither si-NPs nor siPA-NPs had reduced \%FRET in the presence of $2 \mathrm{U} / \mathrm{mL}$ heparin, a dose which was previously used to completely disassemble strictly cationic polyplexes (Figure S5) ${ }^{38}$. At each dose increasing from $10-90 \mathrm{U} / \mathrm{mL}$ heparin, siPA-NPs retained higher \%FRET compared to si-NPs throughout the entire time course (180 $\min$ ) (Figure 4A and S5). Only at the highest heparin dose $(100 \mathrm{U} / \mathrm{mL})$ did siPA-NPs and si-NPs have similar kinetics of reduction in \%FRET over $180 \mathrm{~min}$. An $\mathrm{EC}_{50}$ (indicative of the half maximal concentration of heparin necessary to dissociate polyplexes) was calculated at multiple time points $(30,60,90,120,150$, and $180 \mathrm{~min})$ in order to quantify the dose response of heparin- 
dependent disassembly observed over time. The $\mathrm{EC}_{50}$ of siPA-NPs was $\sim 2$-fold greater than that of si-NPs at each time point analyzed (Table S2), meaning that double the concentration of heparin was required to disassemble siPA-NPs and suggesting that added hydrophobicity of siPA conjugates within siPA-NPs provide increased stability upon exposure to polyanionic challenge such as by heparin sulfates found within the GBM.
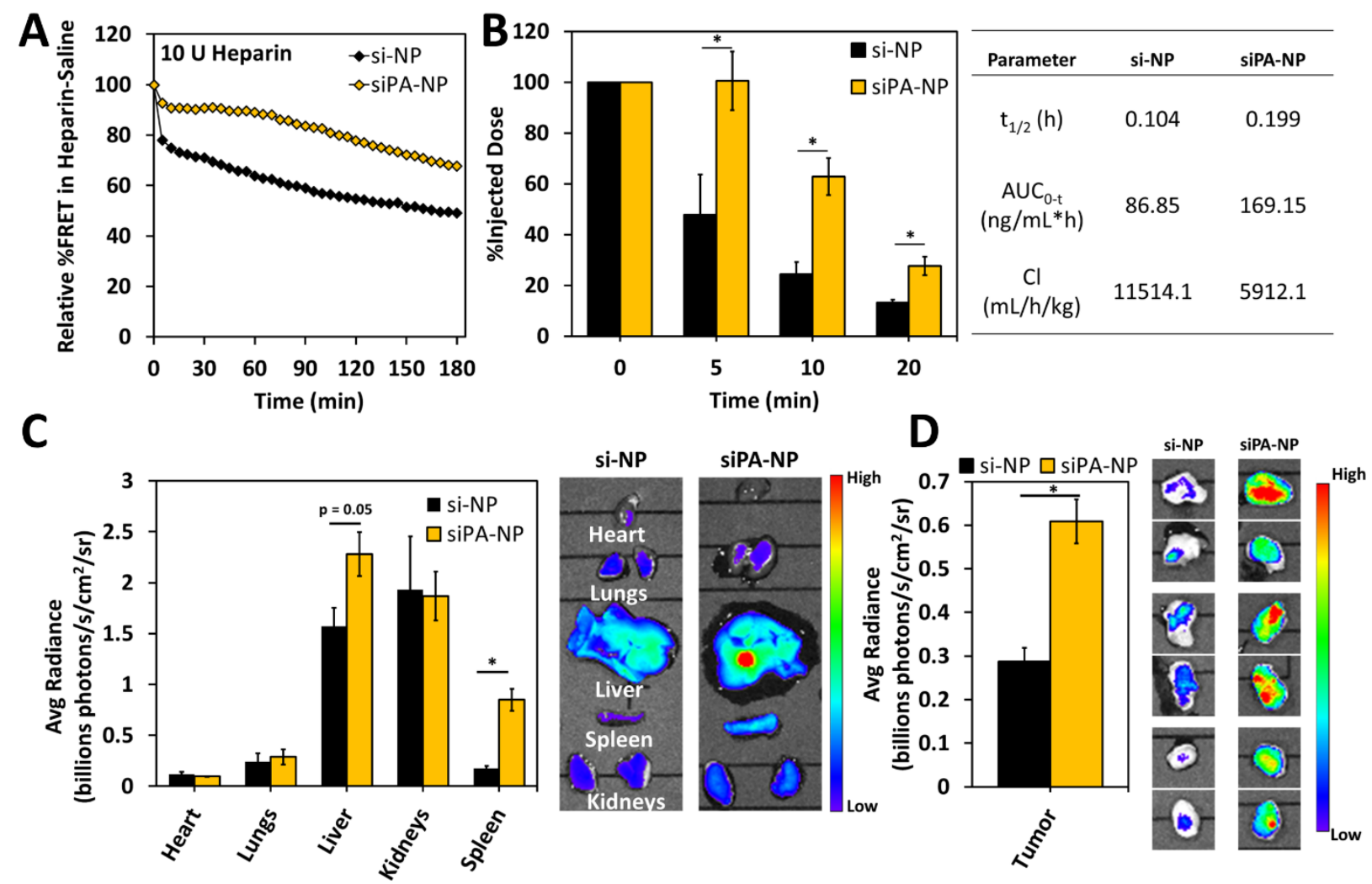

Figure 4: Higher stability of siPA-NPs relative to si-NPs corresponds to greater circulation time and increased accumulation in tumor tissue. A) siPA-NPs are more stable than si-NPs in the presence of heparin, as evaluated by FRET measurements. B) siPA-NPs injected intravenously in mice have a longer circulation half-life than si-NPs. C, D) siPA-NPs accumulate more in tumor tissue and in the MPS organs (liver and spleen) than si-NPs in a mouse tumor model at 20 minutes following tail vein injection.

In blood pharmacokinetics experiments, increased fluorescence was detected within blood samples collected at each time point $(5,10$, and $20 \mathrm{~min})$ from siPA-NPs compared to siNPs (Figure 4B). The calculated circulation half-life of siPA-NPs $(0.199 \mathrm{~h})$ was $\sim 2$-fold greater than si-NPs $(0.104 \mathrm{~h}$ ), resulting in $\sim 2$-fold increase in area under the curve (AUC), and $\sim 2$ fold decrease in blood clearance (CL) (Figure 4B). The observation of increased circulation persistence is especially important due to its correlation with passive tumor accumulation, which was studied in athymic nude mice bearing orthotopic xenografts of MDA-MB-231 triple negative breast cancer cells in the mammary fat pad. Biodistribution in major organs of interest (heart, lungs, liver, kidneys, and spleen) after i.v. administration of $1 \mathrm{mg} / \mathrm{kg}$ nanoparticles (siRNA dose) was comparable between siPA-NPs and si-NPs (Figure 4C). As a result of 
decreased renal clearance and consequent increased exposure to other organs and tissues, the siPA-NPs exhibited higher levels of uptake within MPS organs (liver and spleen) than si-NPs. Importantly, a 2-fold increase in tumor uptake was observed by siPA-NPs (Figure 4D), confirming that increased circulation time of siPA-NPs translated to increased EPR-based passive tumor uptake. Commonly, active targeting ligands such as folic acid, hyaluronic acid, RGD peptide, or transferrin, are used as a strategy to increase tumor uptake and retention after i.v. administration ${ }^{36,60-64}$. Herein, tumor uptake was increased by tuning the core chemistry of polyplexes to increase polyplex stability and circulation time. Thus, it is expected that the addition of appropriate targeting ligands in the future will further increase tumor concentration due to improved tumor retention.

The in-depth studies above thoroughly compare the impact of PA-modified vs. unmodified siRNA with the hydrophobized 50B carrier. Conjugation of alkyl chains similar to PA has also been explored as a strategy to improve pharmacokinetics of therapeutics delivered carrier-free by leveraging lipid binding to serum proteins ${ }^{65}$. A comprehensive evaluation of lipidmodified siRNAs revealed that PA modification is less ideal for eliciting serum protein binding than longer and/or more complex lipids such as cholesterol ${ }^{66}$. Based on this prior work and the need to enhance siRNA activity with an endosomolytic moiety, we incorporated PA-modified siRNA into the core of hydrophobic and endosomolytic siPA-NPs. The improved pharmacokinetics of siPA-NPs over si-NPs is likely due to their enhanced stability from hydrophobic interactions between the PA-modified siRNA and the hydrophobized 50B carrier. To confirm this, we ran a set of control experiments using the fully cationic polymeric carrier 0B. We investigated si-0B-NPs and siPA-OB-NPs in a subset of studies to rule out the possibility that our observations were driven solely by hydrophobization of the siRNA. The degree of siRNA unpackaging in the presence of heparin was evaluated for si-NPs, siPA-NPs, si-0B-NPs, siPA-0B-NPs. In accordance with previous reports si-NPs and siPA-NPs were more stable in response to heparin than si-0B-NPs and siPA-0B-NPs. siPA-NPs showed an additional enhancement in stability relative to si-NPs that was not observed in si-0B-NPs vs. siPA-0B-NPs (Figure S6). Significantly, 0B polyplexes loaded with siPA did not show any increase in blood circulation half-life relative to $0 \mathrm{~B}$ polyplexes loaded with unmodified siRNA, while siPA increased circulation time approximately 2 -fold relative to unmodified siRNA in 50B polyplexes (Figure S7). These data confirm that the hydrophobic interactions between siPA and the BMA of the 50B NP core are critical to the enhanced stability and circulation time observed for siPANPs.

Target Gene Silencing and Acute Toxicity In Vivo. To measure the impact of the improved pharmacokinetics of siPA-NPs on tumor bioactivity, the in vivo bioluminescence was tracked in mice bearing luciferase-expressing MDA-MB-231 orthotopic xenografts of the mammary fat pad after i.v. injection $(1 \mathrm{mg} / \mathrm{kg}$ siRNA dose) on days 17 and 18 after transplantation. The relative luminescence measured by intravital imaging increased steadily over $48 \mathrm{~h}$ (up to 2.5 -fold) in mice administered saline, si-NP scrambled, and siPA-NP scrambled. Mice administered 
luciferase si-NPs did not show a treatment response after the first day, but they showed significant reduction in bioluminescence at 48 hours (after 2 consecutive treatments). The mice administered siPA-NPs showed a treatment response at both $24 \mathrm{~h}$ (after 1 treatment) and 48 hours (after 2 consecutive treatments), with significant decreases in bioluminescence compared to saline and scrambled controls (Figure 5A). Moreover, the bioluminescence of mice administered siPA-NPs did not significantly increase above the baseline measure at 0 hours throughout the time course, indicating complete inhibition of luminescence associated with tumor growth over the $48 \mathrm{~h}$ treatment protocol. These data strongly support the effectiveness of siPA-NPs for tumor target gene silencing after i.v. administration and confirms the functional significance of the increased stability, circulation time, and tumor uptake of siPA-NPs. This increase in efficacy is expected to be impactful for knockdown of pathological genes, with these results suggesting that siPA-NPs would achieve a therapeutic response with a lower dose or fewer administrations relative to si-NPs.
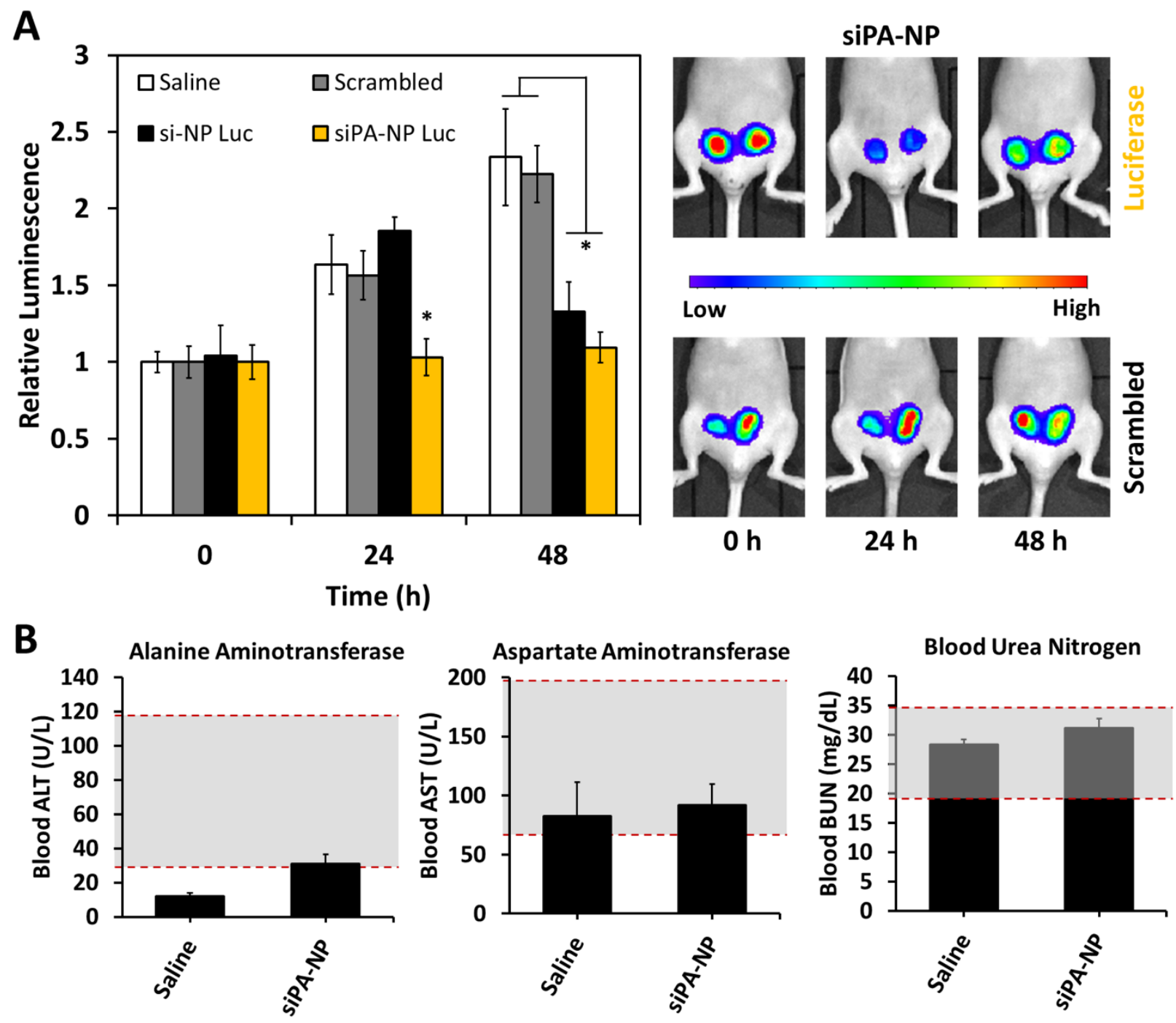

Figure 5: siPA-NPs delivered intravenously silence luciferase in an orthotopic MDA-MB-231 tumor model more effectively than si-NPs and cause no significant liver and kidney toxicity. A) Luciferase silencing, compared to 
scrambled siRNA controls. B) Measurements of liver (ALT and AST) and kidney (BUN) toxicity for si-NP and siPA-NPs $48 \mathrm{~h}$ after treatments.

Because there was significant accumulation of siPA-NPs in the liver and kidneys, ALT and AST (indicators of liver function / toxicity) and BUN (indicator of kidney function / toxicity) were assessed in the treated mice. Blood collected from mice at the time of euthanasia (48 hours after the last treatment) showed that systemic levels of ALT, AST, and BUN were not significantly elevated by the treatment protocol of si-NPs or siPA-NPs utilized. ALT and AST was increased in si-NPs above the mean levels of saline and siPA-NPs, but the increase was not statistically significant (Figure 5B).

\section{$\underline{\text { Conclusions }}$}

The performance of PEG- $b$-p(DMAEMA-co-BMA) "50B", which forms siRNA-loaded NPs with balanced cationic and hydrophobic core content, can be improved through pairing with siPA hydrophobized siRNA. The hydrophobicity of both the polymer and siPA molecule was essential to improved polyplex stability, which can be attributed to increased van der Waals interactions between carrier and cargo. These interactions facilitated more efficient siPA loading into NPs and siPA-NP polyplexes were also more resilient to heparin sulfate-induced destabilization. Increased siPA-NP stability, when compared to our benchmark si-NPs, resulted in increased blood circulation time and EPR-driven passive uptake into orthotopic tumor xenografts after intravenous polyplex injections. The enhanced pharmacokinetics of siPA-NPs translated to increased bioactivity of siRNA, as assessed by target gene silencing of the model gene luciferase within orthotopic triple negative breast cancer (MDA-MB-231) tumors. Our results demonstrate that increasing the strength of associative forces, rather than solely utilizing electrostatic forces that are traditionally leveraged to drive polyplex assembly, can increase both polyplex stability and bioactivity in vivo. The data support continued efforts to stabilize siRNA NP systems to improve pharmacokinetics and pharmacodynamics of siRNA and increase clinical translatability for cancer applications.

\section{$\underline{\text { Acknowledgements }}$}

We are grateful to the National Institutes of Health for financial support (NIH NIBIB R21EB012750 and R01EB019409) for these studies. We are grateful to the National Science Foundation for financial support (NSF CAREER BMAT 1349604), and this material is based upon work supported by the National Science Foundation Graduate Research Fellowship Program under Grants No. 1445191, 1445197, and 0909667. DLS experiments were performed through core instruments provided by the Vanderbilt Institute for Nanoscale Science and Engineering (VINSE). 


\section{$\underline{\text { References }}$}

1. Wang J, Lu Z, Wientjes MG, Au JL. Delivery of sirna therapeutics: Barriers and carriers. The AAPS journal. 2010;12:492-503

2. Gilleron J, Querbes W, Zeigerer A, Borodovsky A, Marsico G, Schubert U, Manygoats K, Seifert S, Andree C, Stoter M, Epstein-Barash H, Zhang L, Koteliansky V, Fitzgerald K, Fava E, Bickle M, Kalaidzidis Y, Akinc A, Maier M, Zerial M. Image-based analysis of lipid nanoparticle-mediated sirna delivery, intracellular trafficking and endosomal escape. Nature biotechnology. 2013;31:638-646

3. Haussecker D. Current issues of rnai therapeutics delivery and development. Journal of controlled release : official journal of the Controlled Release Society. 2014;195:49-54

4. Zuckerman JE, Choi $\mathrm{CH}$, Han $\mathrm{H}$, Davis ME. Polycation-sirna nanoparticles can disassemble at the kidney glomerular basement membrane. Proceedings of the National Academy of Sciences of the United States of America. 2012;109:3137-3142

5. Naeye B, Deschout H, Caveliers V, Descamps B, Braeckmans K, Vanhove C, Demeester J, Lahoutte T, De Smedt SC, Raemdonck K. In vivo disassembly of iv administered sirna matrix nanoparticles at the renal filtration barrier. Biomaterials. 2013;34:2350-2358

6. Pun SH, Davis ME. Development of a nonviral gene delivery vehicle for systemic application. Bioconjugate Chemistry. 2002;13:630-639

7. Convertine AJ, Benoit DS, Duvall CL, Hoffman AS, Stayton PS. Development of a novel endosomolytic diblock copolymer for sirna delivery. Journal of controlled release : official journal of the Controlled Release Society. 2009;133:221-229

8. Oe Y, Christie RJ, Naito M, Low SA, Fukushima S, Toh K, Miura Y, Matsumoto Y, Nishiyama N, Miyata K, Kataoka K. Actively-targeted polyion complex micelles stabilized by cholesterol and disulfide cross-linking for systemic delivery of sirna to solid tumors. Biomaterials. 2014;35:7887-7895

9. Li H, Miteva M, Kirkbride KC, Cheng MJ, Nelson CE, Simpson EM, Gupta MK, Duvall $\mathrm{CL}$, Giorgio TD. Dual mmp7-proximity-activated and folate receptor-targeted nanoparticles for sirna delivery. Biomacromolecules. 2015;16:192-201

10. Yin H, Kanasty RL, Eltoukhy AA, Vegas AJ, Dorkin JR, Anderson DG. Non-viral vectors for gene-based therapy. Nature reviews. Genetics. 2014;15:541-555 
11. Alam MR, Ming X, Fisher M, Lackey JG, Rajeev KG, Manoharan M, Juliano RL. Multivalent cyclic rgd conjugates for targeted delivery of small interfering rna. Bioconjugate chemistry. 2011;22:1673-1681

12. Kubo T, Takei Y, Mihara K, Yanagihara K, Seyama T. Amino-modified and lipidconjugated dicer-substrate sirna enhances rnai efficacy. Bioconjugate chemistry. 2012;23:164-173

13. Jeong JH, Mok, H., Oh, Y., and Park, T.G. Sirna conjugate delivery systems. Bioconjugate Chem. 2009;20:5-14

14. Matsuda S, Keiser K, Nair JK, Charisse K, Manoharan RM, Kretschmer P, Peng CG, A VKi, Kandasamy P, Willoughby JL, Liebow A, Querbes W, Yucius K, Nguyen T, Milstein S, Maier MA, Rajeev KG, Manoharan M. Sirna conjugates carrying sequentially assembled trivalent n-acetylgalactosamine linked through nucleosides elicit robust gene silencing in vivo in hepatocytes. ACS chemical biology. 2015;10:1181-1187

15. Nishina K, Unno T, Uno Y, Kubodera T, Kanouchi T, Mizusawa H, Yokota T. Efficient in vivo delivery of sirna to the liver by conjugation of alpha-tocopherol. Molecular therapy: the journal of the American Society of Gene Therapy. 2008;16:734-740

16. Nelson CE, Kintzing, R. J., Hanna, A., Shannon, J. M., Gupta, M. K., Duvall, C. L. Balancing cationic and hydrophobic content of pegylated sirna polyplexes enhances endosome escape, stability, blood circulation time, and bioactivity in vivo. ACS Nano. 2013;7:8870-8880

17. Poon Z, Chang D, Zhao X, Hammond PT. Layer-by-layer nanoparticles with a phsheddable layer for in vivo targeting of tumor hypoxia. ACS Nano. 2011;5:4284-4292

18. Wei H, Volpatti LR, Sellers DL, Maris DO, Andrews IW, Hemphill AS, Chan LW, Chu DSH, Horner PJ, Pun SH. Dual responsive, stabilized nanoparticles for efficient in vivo plasmid delivery. Angewandte Chemie International Edition. 2013;52:5377-5381

19. Foster AA, Greco CT, Green MD, Epps TH, Sullivan MO. Light-mediated activation of sirna release in diblock copolymer assemblies for controlled gene silencing. Advanced Healthcare Materials. 2015;4:760-770

20. Nakayama T, Butler JS, Sehgal A, Severgnini M, Racie T, Sharman J, Ding F, Morskaya SS, Brodsky J, Tchangov L, Kosovrasti V, Meys M, Nechev L, Wang G, Peng CG, Fang Y, Maier M, Rajeev KG, Li R, Hettinger J, Barros S, Clausen V, Zhang X, Wang Q, Hutabarat R, Dokholyan NV, Wolfrum C, Manoharan M, Kotelianski V, Stoffel M, Sah DW. Harnessing a physiologic mechanism for sirna delivery with mimetic lipoprotein particles. Molecular therapy : the journal of the American Society of Gene Therapy. 2012;20:1582-1589

21. Rajeev KG, Nair JK, Jayaraman M, Charisse K, Taneja N, O'Shea J, Willoughby JL, Yucius K, Nguyen T, Shulga-Morskaya S, Milstein S, Liebow A, Querbes W, Borodovsky A, Fitzgerald K, Maier MA, Manoharan M. Hepatocyte-specific delivery of sirnas conjugated to novel non-nucleosidic trivalent n-acetylgalactosamine elicits robust gene silencing in vivo. Chembiochem : a European journal of chemical biology. 2015;16:903-908

22. Yasuda M, Gan L, Chen B, Kadirvel S, Yu C, Phillips JD, New MI, Liebow A, Fitzgerald K, Querbes W, Desnick RJ. Rnai-mediated silencing of hepatic alas1 effectively prevents and treats the induced acute attacks in acute intermittent porphyria mice. Proceedings of the National Academy of Sciences of the United States of America. 2014;111:7777-7782 
23. Coelho T, Adams D, Silva A, Lozeron P, Hawkins PN, Mant T, Perez J, Chiesa J, Warrington S, Tranter E, Munisamy M, Falzone R, Harrop J, Cehelsky J, Bettencourt BR, Geissler M, Butler JS, Sehgal A, Meyers RE, Chen Q, Borland T, Hutabarat RM, Clausen VA, Alvarez R, Fitzgerald K, Gamba-Vitalo C, Nochur SV, Vaishnaw AK, Sah DW, Gollob JA, Suhr OB. Safety and efficacy of rnai therapy for transthyretin amyloidosis. The New England journal of medicine. 2013;369:819-829

24. Frank-Kamenetsky M, Grefhorst A, Anderson NN, Racie TS, Bramlage B, Akinc A, Butler D, Charisse K, Dorkin R, Fan Y, Gamba-Vitalo C, Hadwiger P, Jayaraman M, John M, Jayaprakash KN, Maier M, Nechev L, Rajeev KG, Read T, Rohl I, Soutschek J, Tan P, Wong J, Wang G, Zimmermann T, de Fougerolles A, Vornlocher HP, Langer R, Anderson DG, Manoharan M, Koteliansky V, Horton JD, Fitzgerald K. Therapeutic rnai targeting pcsk9 acutely lowers plasma cholesterol in rodents and ldl cholesterol in nonhuman primates. Proceedings of the National Academy of Sciences of the United States of America. 2008;105:11915-11920

25. Lammers T, Rizzo LY, Storm G, Kiessling F. Personalized nanomedicine. Clinical Cancer Research. 2012;18:4889-4894

26. Hansen AE, Petersen AL, Henriksen JR, Boerresen B, Rasmussen P, Elema DR, Rosenschöld PMa, Kristensen AT, Kjær A, Andresen TL. Positron emission tomography based elucidation of the enhanced permeability and retention effect in dogs with cancer using copper-64 liposomes. ACS Nano. 2015;9:6985-6995

27. Gabizon A, Shmeeda H, Barenholz Y. Pharmacokinetics of pegylated liposomal doxorubicin. Clinical Pharmacokinetics. 2003;42:419-436

28. Papahadjopoulos D, Allen TM, Gabizon A, Mayhew E, Matthay K, Huang SK, Lee KD, Woodle MC, Lasic DD, Redemann C. Sterically stabilized liposomes: Improvements in pharmacokinetics and antitumor therapeutic efficacy. Proceedings of the National Academy of Sciences. 1991;88:11460-11464

29. Torchilin V. Tumor delivery of macromolecular drugs based on the epr effect. Adv Drug Deliv Rev. 2011;63:131-135

30. Maeda H, Nakamura H, Fang J. The epr effect for macromolecular drug delivery to solid tumors: Improvement of tumor uptake, lowering of systemic toxicity, and distinct tumor imaging in vivo. Adv Drug Deliv Rev. 2013;65:71-79

31. Venkataraman S, Ong WL, Ong ZY, Joachim Loo SC, Rachel Ee PL, Yang YY. The role of peg architecture and molecular weight in the gene transfection performance of pegylated poly(dimethylaminoethyl methacrylate) based cationic polymers. Biomaterials. 2011;32:2369-2378

32. Mishra S, Webster P, Davis ME. Pegylation significantly affects cellular uptake and intracellular trafficking of non-viral gene delivery particles. European Journal of Cell Biology. 2004;83:97-111

33. Sato A, Choi SW, Hirai M, Yamayoshi A, Moriyama R, Yamano T, Takagi M, Kano A, Shimamoto A, Maruyama A. Polymer brush-stabilized polyplex for a sirna carrier with long circulatory half-life. Journal of Controlled Release. 2007;122:209-216

34. Miteva M, Kirkbride KC, Kilchrist KV, Werfel TA, Li H, Nelson CE, Gupta MK, Giorgio TD, Duvall CL. Tuning pegylation of mixed micelles to overcome intracellular and systemic sirna delivery barriers. Biomaterials. 2015;38:97-107

35. Merkel OM, Librizzi D, Pfestroff A, Schurrat T, Buyens K, Sanders NN, De Smedt SC, Béhé M, Kissel T. Stability of sirna polyplexes from poly(ethylenimine) and 
poly(ethylenimine)-g-poly(ethylene glycol) under in vivo conditions: Effects on pharmacokinetics and biodistribution measured by fluorescence fluctuation spectroscopy and single photon emission computed tomography (spect) imaging. Journal of Controlled Release. 2009;138:148-159

36. Deng ZJ, Morton SW, Ben-Akiva E, Dreaden EC, Shopsowitz KE, Hammond PT. Layerby-layer nanoparticles for systemic codelivery of an anticancer drug and sirna for potential triple-negative breast cancer treatment. ACS Nano. 2013; 7:9571-9584

37. Zhu X, Xu Y, Solis LM, Tao W, Wang L, Behrens C, Xu X, Zhao L, Liu D, Wu J, Zhang N, Wistuba II, Farokhzad OC, Zetter BR, Shi J. Long-circulating sirna nanoparticles for validating prohibitin1-targeted non-small cell lung cancer treatment. Proceedings of the National Academy of Sciences. 2015;112:7779-7784

38. Nelson CE, Kintzing JR, Hanna A, Shannon JM, Gupta MK, Duvall CL. Balancing cationic and hydrophobic content of pegylated sirna polyplexes enhances endosome escape, stability, blood circulation time, and bioactivity in vivo. ACS Nano. 2013;7:88708880

39. Kubo T, Yanagihara K, Sato Y, Nishimura Y, Kondo S, Seyama T. Gene-silencing potency of symmetric and asymmetric lipid-conjugated sirnas and its correlation with dicer recognition. Bioconjugate chemistry. 2013;24:2045-2057

40. Ambardekar VV, Han HY, Varney ML, Vinogradov SV, Singh RK, Vetro JA. The modification of sirna with 3' cholesterol to increase nuclease protection and suppression of native mrna by select sirna polyplexes. Biomaterials. 2011;32:1404-1411

41. Uno Y, Piao W, Miyata K, Nishina K, Mizusawa H, Yokota T. High-density lipoprotein facilitates in vivo delivery of alpha-tocopherol-conjugated short-interfering rna to the brain. Human gene therapy. 2011;22:711-719

42. Sarett SM, Kilchrist KV, Miteva M, Duvall CL. Conjugation of palmitic acid improves potency and longevity of sirna delivered via endosomolytic polymer nanoparticles. Journal of biomedical materials research. Part A. 2015

43. Oba M, Miyata K, Osada K, Christie RJ, Sanjoh M, Li W, Fukushima S, Ishii T, Kano MR, Nishiyama N, Koyama H, Kataoka K. Polyplex micelles prepared from omegacholesteryl peg-polycation block copolymers for systemic gene delivery. Biomaterials. 2011;32:652-663

44. Falamarzian A, Aliabadi HM, Molavi O, Seubert JM, Lai R, Uludag H, Lavasanifar A. Effective down-regulation of signal transducer and activator of transcription 3 (stat3) by polyplexes of sirna and lipid-substituted polyethyleneimine for sensitization of breast tumor cells to conventional chemotherapy. Journal of biomedical materials research. Part A. 2014; 102:3216-3228

45. Wei H, Volpatti LR, Sellers DL, Maris DO, Andrews IW, Hemphill AS, Chan LW, Chu DS, Horner PJ, Pun SH. Dual responsive, stabilized nanoparticles for efficient in vivo plasmid delivery. Angewandte Chemie. 2013;52:5377-5381

46. Kim J, Sunshine, J. C., Green, J. J. Differential polymer structure tunes mechanism of cellular uptake and transfection routes of poly( $\beta$-amino ester) polyplexes in human breast cancer cells. Bioconjugate chemistry. 2014;25

47. Sunshine JC, Akanda MI, Li D, Kozielski KL, Green JJ. Effects of base polymer hydrophobicity and end-group modification on polymeric gene delivery. Biomacromolecules. 2011;12:3592-3600 
48. Bishop CJ, Abubaker-Sharif B, Guiriba T, Tzeng SY, Green JJ. Gene delivery polymer structure-function relationships elucidated via principal component analysis. Chemical Communications. 2015;51:12134-12137

49. Chiefari J, Chong YK, Ercole F, Krstina J, Jeffery J, Le TPT, Mayadunne RTA, Meijs GF, Moad CL, Moad G, Rizzardo E, Thang SH. Living free-radical polymerization by reversible addition-fragmentation chain transfer: The raft process. Macromolecules. 1998;31:5559-5562

50. Liu H, Li Y, Mozhi A, Zhang L, Liu Y, Xu X, Xing J, Liang X, Ma G, Yang J, Zhang X. Sirna-phospholipid conjugates for gene and drug delivery in cancer treatment. Biomaterials. 2014;35:6519-6533

51. He C, Hu Y, Yin L, Tang C, Yin C. Effects of particle size and surface charge on cellular uptake and biodistribution of polymeric nanoparticles. Biomaterials. 2010;31:3657-3666

52. Tang L, Fan TM, Borst LB, Cheng J. Synthesis and biological response of size-specific, monodisperse drug-silica nanoconjugates. ACS Nano. 2012;6:3954-3966

53. Gratton SEA, Ropp PA, Pohlhaus PD, Luft JC, Madden VJ, Napier ME, DeSimone JM. The effect of particle design on cellular internalization pathways. Proceedings of the National Academy of Sciences. 2008;105:11613-11618

54. Perry JL, Reuter KG, Kai MP, Herlihy KP, Jones SW, Luft JC, Napier M, Bear JE, DeSimone JM. Pegylated print nanoparticles: The impact of peg density on protein binding, macrophage association, biodistribution, and pharmacokinetics. Nano Letters. 2012;12:5304-5310

55. Anselmo AC, Zhang M, Kumar S, Vogus DR, Menegatti S, Helgeson ME, Mitragotri S. Elasticity of nanoparticles influences their blood circulation, phagocytosis, endocytosis, and targeting. ACS Nano. 2015;9:3169-3177

56. Nam HY, Kwon SM, Chung H, Lee S-Y, Kwon S-H, Jeon H, Kim Y, Park JH, Kim J, Her S, Oh Y-K, Kwon IC, Kim K, Jeong SY. Cellular uptake mechanism and intracellular fate of hydrophobically modified glycol chitosan nanoparticles. Journal of Controlled Release. 2009;135:259-267

57. Sahay G, Querbes W, Alabi C, Eltoukhy A, Sarkar S, Zurenko C, Karagiannis E, Love K, Chen D, Zoncu R, Buganim Y, Schroeder A, Langer R, Anderson DG. Efficiency of sirna delivery by lipid nanoparticles is limited by endocytic recycling. Nat Biotech. 2013;31:653-658

58. Werfel TA, Swain C, Nelson CE, Kilchrist KV, Evans BC, Miteva M, Duvall CL. Hydrolytic charge-reversal of pegylated polyplexes enhances intracellular un-packaging and activity of sirna. Journal of Biomedical Materials Research Part A. 2016;104:917927

59. Evans BC HK, Osgood MJ, Voskresensky I, Dmowska J, Kilchrist KV, Brophy CM, Duvall, CL. Mk2 inhibitory peptide delivered in nanopolyplexes prevents vascular graft intimal hyperplasia. Science Translational Medicine. 2015;7

60. Gabizon A, Shmeeda H, Horowitz AT, Zalipsky S. Tumor cell targeting of liposomeentrapped drugs with phospholipid-anchored folic acid-peg conjugates. Advanced drug delivery reviews. 2004;56:1177-1192

61. Choi KY, Chung H, Min KH, Yoon HY, Kim K, Park JH, Kwon IC, Jeong SY. Selfassembled hyaluronic acid nanoparticles for active tumor targeting. Biomaterials. 2010;31:106-114 
62. Arap W, Pasqualini R, Ruoslahti E. Cancer treatment by targeted drug delivery to tumor vasculature in a mouse model. Science. 1998;279:377-380

63. Bellocq NC, Pun SH, Jensen GS, Davis ME. Transferrin-containing, cyclodextrin polymer-based particles for tumor-targeted gene delivery. Bioconjugate chemistry. 2003;14:1122-1132

64. Davis ME, Zuckerman JE, Choi CHJ, Seligson D, Tolcher A, Alabi CA, Yen Y, Heidel $\mathrm{JD}$, Ribas A. Evidence of rnai in humans from systemically administered sirna via targeted nanoparticles. Nature. 2010;464:1067-1070

65. Pollaro L, Heinis C. Strategies to prolong the plasma residence time of peptide drugs. MedChemComm. 2010;1:319

66. Wolfrum C, Shi S, Jayaprakash KN, Jayaraman M, Wang G, Pandey RK, Rajeev KG, Nakayama T, Charrise K, Ndungo EM, Zimmermann T, Koteliansky V, Manoharan M, Stoffel M. Mechanisms and optimization of in vivo delivery of lipophilic sirnas. Nature biotechnology. 2007;25:1149-1157 